\title{
Multi-Objective Optimization of Wireless Information and Power Transfer in Multiuser OFDMA Systems
}

\author{
Hsin-Jui Chou*, Ronald Y. Chang*, and Jen-Ming $\mathrm{Wu}^{\dagger}$ \\ ${ }^{*}$ Research Center for Information Technology Innovation, Academia Sinica, Taipei, Taiwan \\ $\dagger$ Institute of Communications Engineering, National Tsing Hua University, Hsinchu, Taiwan \\ Email: \{hjchou, rchang\}@ citi.sinica.edu.tw, jmwu@ee.nthu.edu.tw
}

\begin{abstract}
This paper studies joint subchannel allocation, power allocation, and beamforming for simultaneous wireless information and power transfer (SWIPT) in multiuser downlink orthogonal frequency-division multiple access (OFDMA) systems. We formulate a multi-objective optimization (MOO) problem where the objectives are to maximize both the information rate and the harvested power for all users in the system. We approach the MOO problem with two proposed methods, i.e., semidefinite relaxation-based weighted aggregation (SDR-WA) and multiobjective genetic algorithm (MOGA). Simulation compares the achievable Pareto optimal solution set yielded by these methods, and illustrates the tradeoffs of the sum information rate vs. the sum harvested power in the system.

Index Terms-Energy harvesting communications, simultaneous wireless information and power transfer (SWIPT), multiobjective optimization (MOO), orthogonal frequency-division multiple access (OFDMA).
\end{abstract}

\section{INTRODUCTION}

The development of device-to-device (D2D) communication and Internet of things (IoT) and of everything (IoE) has significantly increased the demand of communication bandwidth. Meanwhile, since most of the devices (handheld devices, sensor nodes, etc.) in these networks are battery-powered, the demand of a higher battery capacity has also increased [1], [2]. The increase in battery capacity, however, fails to meet the ever-increasing energy demand [3]. It is therefore imperative to seek a more reliable and accessible alternative energy source for the mobile devices. The concept of simultaneous wireless information and power transfer (SWIPT) [3]-[15], which represents harvesting power from the electromagnetic waves used for transmission of information, has emerged as a viable solution to the bandwidth and energy shortage problem.

In single user environments, SWIPT focuses on the design of time-switching or power-splitting to balance the information and energy (power) tradeoff [5]-[9]. In multiuser environments, SWIPT focuses on the resource allocation (channel allocation, transmit power allocation, etc.) among different users for information or energy transmission, such that some

This work was supported in part by the Ministry of Science and Technology, Taiwan, under Grants MOST 103-2221-E-001-027, MOST 103-2218-E-007020, and MOST 104-2628-E-001-002-MY2. quality-of-service (QoS) criteria (throughput, harvested power, packet loss, fairness, etc.) are met [10]-[15]. This renders a multi-objective optimization (MOO) problem with multiple, often conflicting objectives. Existing work studying SWIPT has considered reformulating an MOO into a single objective optimization (SOO) problem. The reformulation is based on a weighted aggregation of the multiple objectives [10], [12], or a transformation of some objectives into constraints [10]-[15]. This approach has several limitations: 1) the conversion into SOO requires prior knowledge about the relative importance of each objective; even so, the accurate and precise selection on the weights could be quite difficult in practice, 2) the formulation of SOO may lose some perspectives of tradeoff among different objectives, and 3) the transformation of some objectives into constraints may produce improper (unattainable) constraints, which could result in an infeasible problem [16]-[18].

In this work, we consider the joint design of subchannel allocation, power allocation, and precoding (beamforming) in a multiuser downlink orthogonal frequency-division multiple access (OFDMA) system for SWIPT. The objectives in this MOO problem are to maximize the information rate and the harvested power for all users. As typical in most MOO problems, there is no global optimal solution to this problem, as different objectives are conflicting with each other. Moreover, jointly optimizing subchannel allocation, power allocation, and precoding matrices is a non-convex problem, and it is challenging to solve since the correlation between the variables is hard to be formulated [19]. Thus, we aim to find the Pareto front [16], [17], which represents the "best achievable" objective values from a system-wide perspective by taking into account the tradeoff among different objectives. We propose two methods to solve the considered MOO problem, namely, the semidefinite relaxation-based weighted aggregation (SDR-WA) method and the multi-objective genetic algorithm (MOGA). SDR-WA applies the SDR technique to the SOO problem reformulated from the MOO problem, where the SOO problem can be constructed by any possible linear combination of all objectives and is guaranteed to be a feasible problem. MOGA emulates the biological evolution 


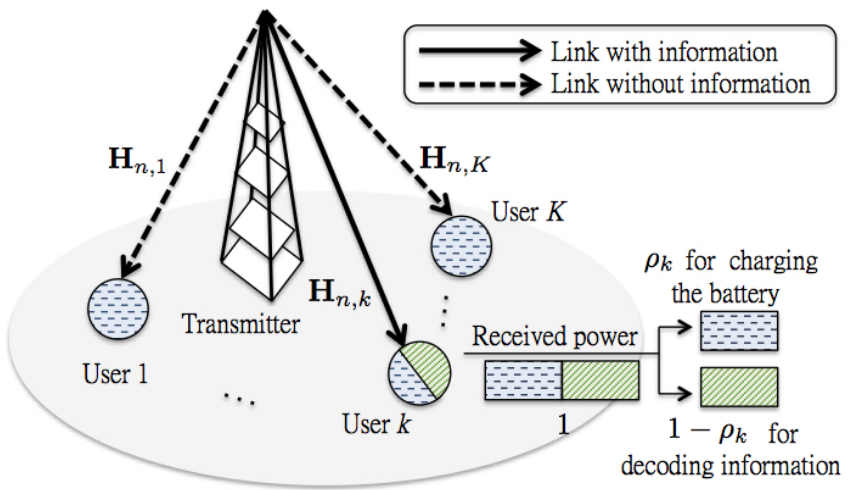

Fig. 1. A downlink multiuser OFDMA system with $N$ subchannels and $K$ users for simultaneous information and power transfer. The transmitter (base station) uses subchannel $n$ to transmit information to user $k$. For user $k, 1-\rho_{k}$ fraction of its received power is used to decode information and $\rho_{k}$ fraction is used to charge its own battery. For other unintended user $l(\forall l \neq k), \rho_{l}$ fraction of its received power is used to charge its own battery.

and screening process to find potential solutions by optimizing all objectives simultaneously. Simulation demonstrates the validity and convergence of the proposed methods, and compares their performance in different system configurations.

The outline of the paper is as follows. Sec. II describes the system model. Sec. III introduces the Pareto optimality concept and describes the considered MOO problem. Sec. IV presents SDR-WA and MOGA methods for solving the MOO problem. Sec. V presents performance results and discussions. Finally, Sec. VI concludes the paper.

Notations: Boldface lowercase letters denote vectors and boldface uppercase letters denote matrices. Calligraphic letters denote sets, and $|\mathcal{A}|$ is the cardinality of set $\mathcal{A}$. $\mathbf{A}=$ $\left\{\mathbf{A}_{l}\right\}_{l=1, \ldots, K}$ represents that matrix $\mathbf{A}$ is a horizontal concatenation of matrices $\mathbf{A}_{1}, \ldots, \mathbf{A}_{K} . \mathbf{A} \succeq 0$ means that matrix $\mathbf{A}$ is positive semidefinite. $\operatorname{tr}(\mathbf{A})$ denotes the trace of matrix A. $(\cdot)^{\top}$ denotes the transpose operation. $\|\cdot\|$ denotes the $l_{2}$ norm of a vector/matrix. $\mathbb{R}$ denotes the set of real numbers. $(a)^{+} \triangleq \max \{a, 0\}$ for some $a \in \mathbb{R}$.

\section{SySTEM MODEL}

We consider a downlink multiuser OFDMA system where a single transmitter (with $M$ antennas) serves $K$ users (each with a single antenna) on $N$ subchannels, as illustrated in Fig. 1. Each subchannel can be allocated to at most one user for information transmission, although all users can observe signals (intended or unintended) transmitted on all subchannels for energy harvesting. Each user may be allocated with multiple subchannels. Let $\alpha_{n, k}=1$ if subchannel $n$ is allocated to user $k$ and $\alpha_{n, k}=0$ otherwise, and $\sum_{k=1}^{K} \alpha_{n, k}=1, \forall n$. Each user adopts a power splitting scheme [10] such that $\rho_{k}$ fraction of the received power at user $k$ is used to charge its battery and $1-\rho_{k}$ fraction is used to decode information. In this work, the power splitting ratio $\rho_{k}$ is a given value and assumed identical for all subchannels allocated to user $k$. All transmitter-user channels are assumed independent and identically distributed (i.i.d.) over $K$ users and $N$ subchannels, and are perfectly known at all nodes.

The received signal at user $k$ on subchannel $n$ is given by

$$
y_{n, k}=p_{n} \mathbf{H}_{n, k} \mathbf{v}_{n} s_{n}+z_{n, k}
$$

where $p_{n}$ is the power allocated on subchannel $n$ with a total power constraint $\sum_{n=1}^{N} p_{n} \leq P ; \mathbf{H}_{n, k} \in \mathbb{R}^{1 \times M}$ is the channel between the transmitter and user $k$ on subchannel $n$, each element following a Gaussian distribution with zero mean and variance $\sigma_{h}^{2} ; s_{n} \in \mathbb{R}$ is the transmit signal on subchannel $n$ for some intended user, with $\mathrm{E}\left[\left\|s_{n}\right\|^{2}\right]=1 ; \mathbf{v}_{n} \in \mathbb{R}^{M \times 1}$ is the corresponding precoding vector with $\left\|\mathbf{v}_{n}\right\|^{2}=1 ; z_{n, k} \in \mathbb{R}$ is the additive white Gaussian noise (AWGN) with zero mean and variance $\sigma_{z}^{2}$.

Information rate for user $k$ : Suppose that subchannel $n$ is allocated to user $k$, i.e., $\alpha_{n, k}=1$ and $\alpha_{n, l}=0, \forall l \neq k$. Then, the information rate of user $k$ on subchannel $n$ can be expressed as

$$
R_{n, k}=\log _{2}\left(1+\left(1-\rho_{k}\right) \frac{p_{n}}{\sigma_{z}^{2}}\left\|\mathbf{H}_{n, k} \mathbf{v}_{n}\right\|^{2}\right) .
$$

The total information rate of user $k$ is

$$
R_{k}=\sum_{n=1}^{N} \alpha_{n, k} R_{n, k} .
$$

Harvested power for user $k$ : A user could harvest the electromagnetic power on subchannel $n$ regardless of whether the transmission on subchannel $n$ is intended for this user or not. The harvested power for user $k$ from the radio wave transmission on subchannel $n$ is given by

$$
\varepsilon_{n, k}=\rho_{k} p_{n}\left\|\mathbf{H}_{n, k} \mathbf{v}_{n}\right\|^{2}
$$

where we have assumed perfect efficiency in the conversion from the radio power to the battery power and thus the factor associated with efficiency loss in power transformation is omitted. The total harvested power of user $k$ is

$$
\varepsilon_{k}=\sum_{n=1}^{N} \varepsilon_{n, k} .
$$

\section{PROBLEM DESCRIPTION}

We aim to find a subchannel allocation scheme (i.e., $\alpha_{n, k}$ ), a power allocation scheme (i.e., $p_{n}$ ), and a precoding scheme (i.e., $\mathbf{v}_{n}$ ) such that the harvested power and the information rate are maximized for all users. This renders a multi-objective optimization (MOO) problem. Unlike single-objective optimization (SOO), in MOO a global optimal solution typically does not exist, because the multiple objectives may be conflicting with one another. However, we may define optimality of a solution in the sense that it is no worse than any other solution in all objectives of the MOO problem. Formally, we have the following definitions [16], [17].

Definition 1 (Pareto Dominance): For the optimization of $Q$ objectives $f_{1}, \ldots, f_{Q}$ in a maximization context, a feasible solution $\mathbf{a}$ is said to dominate another feasible solution $\mathbf{b}$ if 
and only if $f_{i}(\mathbf{a}) \geq f_{i}(\mathbf{b}), \forall i \in\{1, \ldots, Q\}$ and there exists $j \in\{1, \ldots, Q\}$ such that $f_{j}(\mathbf{a})>f_{j}(\mathbf{b})$.

Definition 2 (Pareto Optimality): A feasible solution a is said to be Pareto optimal if and only if there exists no other feasible solution that dominates it. The set of all Pareto optimal solutions is called the Pareto optimal set and the corresponding objectives are called the Pareto front.

Our MOO problem is formulated mathematically as follows, with $2 K$ objectives corresponding to the total harvested power and total information rate of all $K$ users, i.e., $f_{1, k}(\mathcal{P}, \mathcal{V}) \triangleq$ $\varepsilon_{k}, \forall k$ and $f_{2, k}(\mathcal{A}, \mathcal{P}, \mathcal{V}) \triangleq R_{k}, \forall k$ :

$$
\begin{aligned}
& \mathbf{P}_{1}: \max _{\mathcal{A}, \mathcal{P}, \mathcal{V}}( f_{1,1}(\mathcal{P}, \mathcal{V}), \ldots, f_{1, K}(\mathcal{P}, \mathcal{V}), \\
&\left.f_{2,1}(\mathcal{A}, \mathcal{P}, \mathcal{V}), \ldots, f_{2, K}(\mathcal{A}, \mathcal{P}, \mathcal{V})\right)
\end{aligned}
$$

where

$$
\begin{aligned}
& \mathcal{A}=\left\{\left\{\alpha_{n, k}\right\}_{\substack{n=1, \ldots, N \\
k=1, \ldots, K}} \mid \alpha_{n, k} \in\{0,1\}, \sum_{k=1}^{K} \alpha_{n, k}=1\right\} \\
& \mathcal{P}=\left\{\begin{array}{l|l}
\left\{p_{n}\right\}_{n=1, \ldots, N} & \sum_{n=1}^{N} p_{n} \leq P
\end{array}\right\} \\
& \mathcal{V}=\left\{\left\{\mathbf{v}_{n}\right\}_{n=1, \ldots, N} \mid \mathbf{v}_{n} \in \mathbb{R}^{M \times 1},\left\|\mathbf{v}_{n}\right\|^{2}=1\right\} .
\end{aligned}
$$

Our objective is to find the Pareto optimal set for problem $\mathbf{P}_{1}$. This problem is challenging to solve since: 1) $f_{2, k}(\mathcal{A}, \mathcal{P}, \mathcal{V})$ is nonconvex for variables $\mathbf{v}_{n}$ due to the logarithmic-quadratic formulation, 2) the binary integer programming on $\mathcal{A}$ is NPhard, and 3) variables $\alpha_{n, k}, p_{n}$, and $\mathbf{v}_{n}$ cannot be solved independently due to the coupling relationships of $f_{1, k}(\mathcal{P}, \mathcal{V})$ and $f_{2, k}(\mathcal{A}, \mathcal{P}, \mathcal{V}), \forall k$.

\section{The Proposed Solution Methods}

We propose two methods to find the Pareto optimal set and the corresponding Pareto front for problem $\mathbf{P}_{1}$ here, and compare them in Sec. V:

- Semidefinite relaxation-based weighted aggregation (SDR-WA): In this method, the MOO problem is first reformulated into an SOO problem by a linearly weighted combination of the objectives. Then, the SOO problem is solved using the SDR technique. By repeating the SDR-WA process for different weighted combinations of the objectives and solving the resulted SOO, we can obtain a result that approximates the Pareto front. The SDR-WA can be conveniently implemented when there is some a priori information about the weights (e.g., the relative importance of each objective, or the specification of the target values). However, the obtained front may not be globally non-dominated due to the loss of optimality in the relaxation and the alternating maximization process.

- Multi-objective genetic algorithm (MOGA): This method finds potential solutions by optimizing all objectives simultaneously in a single iteration. In contrast to the SDR-WA method, MOGA considers a wider range of tradeoffs between objectives without the need of a priori information and guides the search of solutions toward the Pareto optimal set. However, this method may require a large number of iterations and diversities to prevent premature convergence.

The detailed operation of the two methods is described as follows.

\section{A. Semidefinite Relaxation-based Weighted Aggregation (SDR-WA)}

In this method, problem $\mathbf{P}_{1}$ is first converted to an SOO problem $\mathbf{P}_{2}$ by linearly combining all objectives into one objective, i.e.,

$$
\begin{aligned}
\mathbf{P}_{2}: \max _{\mathcal{A}, \mathcal{P}, \mathcal{V}} & \sum_{k=1}^{K} \beta_{1, k} f_{1, k}(\mathcal{P}, \mathcal{V})+\sum_{k=1}^{K} \beta_{2, k} f_{2, k}(\mathcal{A}, \mathcal{P}, \mathcal{V}) \\
\text { s.t. } & \sum_{i=1}^{2} \sum_{k=1}^{K} \beta_{i, k}=1 \\
& \beta_{1, k} \geq 0, \beta_{2, k} \geq 0, \forall k
\end{aligned}
$$

where $\beta_{i, k}$ is the combining weight of objective $f_{i, j}$, which may depend on some a priori information. Note that the optimal values of $\beta_{i, k}$ cannot be determined within the SOO process. Problem $\mathbf{P}_{2}$ over variables $\mathcal{A}, \mathcal{P}$, and $\mathcal{V}$ can be solved via an alternating maximization (or alternating minimization) [20], i.e., optimizing the objective function for one variable while fixing all other variables, and then alternating the variables, as follows.

Finding $\mathcal{V}$ : It is observed that in problem $\mathbf{P}_{2}$, the precoding vectors $\mathcal{V}$ can be solved independently for different subchannels with given $\mathcal{A}$ and $\mathcal{P}$. That is, the objective in problem $\mathbf{P}_{2}$ can be reformulated as $\sum_{n=1}^{N} \max _{\mathbf{v}_{n}} \sum_{k=1}^{K}\left(\beta_{1, k} \varepsilon_{n, k}+\right.$ $\left.\beta_{2, k} \alpha_{n, k} R_{n, k}\right)$. Specifically, for some subchannel $n$ with user allocation $\alpha_{n, k}=1$ and power allocation $p_{n}$, the precoding vector $\mathbf{v}_{n}$ is designed by solving

$$
\begin{aligned}
\max _{\mathbf{v}_{n} \in \mathcal{V}} & \left(\left(\sum_{l=1}^{K} \beta_{1, l} \rho_{l} p_{n}\left\|\mathbf{H}_{n, l} \mathbf{v}_{n}\right\|^{2}\right)\right. \\
& \left.+\beta_{2, k} \log \left(1+\left(1-\rho_{k}\right) \frac{p_{n}}{\sigma_{z}^{2}}\left\|\mathbf{H}_{n, k} \mathbf{v}_{n}\right\|^{2}\right)\right) .
\end{aligned}
$$

The problem in (9) is an NP-hard problem due to the nonconvexity of the second term in the objective. Thus, we resort to the semidefinite relaxation (SDR) technique [21] by converting the problem into a convex semidefinite programming problem through the relaxation of the quadratic form. Let $\mathbf{V}=\mathbf{v}_{n} \mathbf{v}_{n}^{\top}$. Then, the problem in (9) is reformulated into the following convex problem:

$$
\begin{aligned}
\mathbf{P}_{3}: \quad \lambda_{n, k} \triangleq \max _{\mathbf{V}} & \operatorname{tr}\left(\mathbf{C}_{1} \mathbf{V}\right)+\beta_{2, k} \log \left(1+\operatorname{tr}\left(\mathbf{C}_{2} \mathbf{V}\right)\right) \\
\text { s.t. } & \operatorname{tr}(\mathbf{V}) \leq 1 \\
& \mathbf{V} \succeq 0
\end{aligned}
$$

where $\lambda_{n, k}$ is defined as the optimal solution of problem $\mathbf{P}_{3}, \quad \mathbf{C}_{1}=\sum_{l=1}^{K} \beta_{1, l} \rho_{l} p_{n} \mathbf{H}_{n, l}^{\top} \mathbf{H}_{n, l}$, and $\mathbf{C}_{2}=$ 
$\frac{\left(1-\rho_{k}\right) p_{n}}{\sigma^{2}} \mathbf{H}_{n, k}^{\top} \mathbf{H}_{n, k}$. Problem $\mathbf{P}_{3}$ can be solved using developed techniques such as the interior-point method [22] or the Lagrangian method [23].

Finding $\mathcal{A}$ : By repeatedly assigning subchannel $n$ ( $n=$ $1, \ldots, N)$ to different users, we have $\lambda_{n, 1}, \ldots, \lambda_{n, K}$ after solving problem $\mathbf{P}_{3}$. In order to maximize the objective in problem $\mathbf{P}_{2}$, the subchannel $n$ should be assigned to user $k^{*}$ where $k^{*}=\arg \max _{k} \lambda_{n, k}$, which gives $\alpha_{n, k^{*}}=1$ and $\alpha_{n, k}=0, \forall k \neq k^{*}$.

Finding $\mathcal{P}$ : With given $\mathcal{V}$ and $\mathcal{A}$, the optimal power allocation for each subchannel that maximizes the objective in problem $\mathbf{P}_{2}$ is the solution to the following problem: $\max _{\mathcal{P}} \sum_{n=1}^{N} \sum_{k=1}^{K}\left(\beta_{1, k} \varepsilon_{n, k}+\beta_{2, k} \alpha_{n, k} R_{n, k}\right)$, which is jointly concave in the powers $\left(p_{n}\right)$ and can be solved using Lagrangian methods. Consider the Lagrangian

$$
\begin{aligned}
\mathcal{L} & \left(\lambda, p_{1}, \ldots, p_{N}\right) \\
& =\sum_{n=1}^{N}\left(p_{n}\left(c_{n}-\mu\right)+\sum_{k=1}^{K} \beta_{2, k} \alpha_{n, k} \log \left(1+p_{n} \Delta_{n, k}\right)\right)
\end{aligned}
$$

where $\mu$ is the Lagrange multiplier, $\Delta_{n, k}=\frac{1-\rho_{k}}{\sigma_{z}^{2}}\left\|\mathbf{H}_{n, k} \mathbf{v}_{n}\right\|^{2}$, and $c_{n}=\sum_{k=1}^{K} \beta_{1, k} \rho_{k}\left\|\mathbf{H}_{n, k} \mathbf{v}_{n}\right\|^{2}$. By using the KarushKuhn-Tucker (KKT) condition for the optimality of $p_{n}$, i.e., $\frac{\partial \mathcal{L}}{\partial p_{n}}=0$ if $p_{n}>0$ and $\frac{\partial \mathcal{L}}{\partial p_{n}} \leq 0$ if $p_{n}=0$, the power allocation for subchannel $n$ can be expressed as

$$
p_{n}=\sum_{k=1}^{K} \alpha_{n, k}\left(\frac{\beta_{2, k}}{\left(\mu-c_{n}\right) \ln 2}-\frac{1}{\Delta_{n, k}}\right)^{+}
$$

with the Lagrange multiplier $\mu$ chosen such that the power constraint is met, i.e., $\sum_{n=1}^{N} p_{n}=P$.

The procedure of SDR-WA is summarized in Algorithm 1.

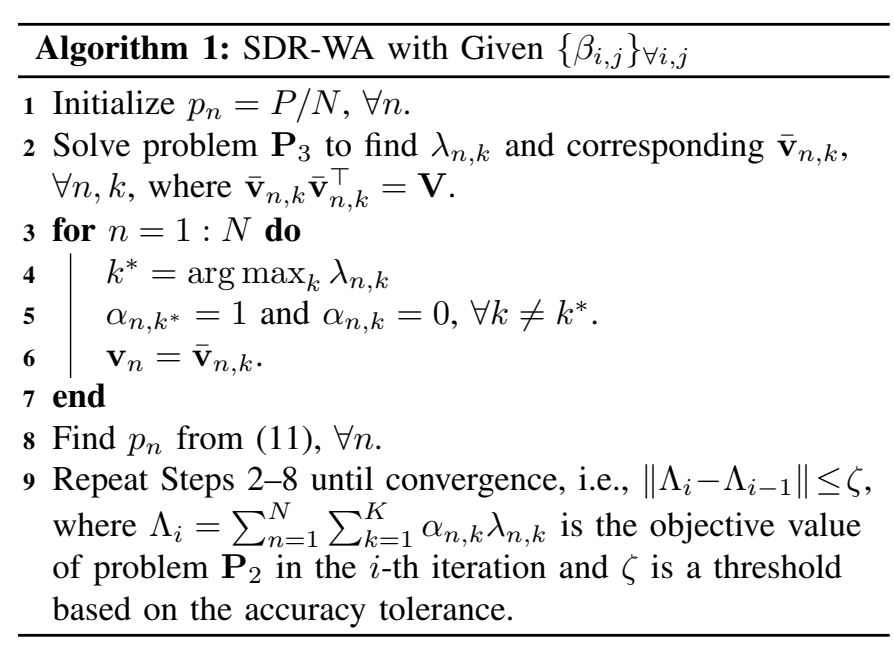

\section{B. Multi-Objective Genetic Algorithm (MOGA)}

MOGA emulates the biological evolution process, where a population of chromosomes (or individuals) representing different solutions of MOO is repeatedly evolving and screened toward the Pareto optimal set [16]. MOGA is comprised of six stages, i.e., initialization, evolution, proliferation, mutation, evaluation, and selection. The design procedure is described as follows and summarized in Algorithm 2.

Initialization: A population $\Omega=\left\{\mathcal{W}_{1}, \ldots, \mathcal{W}_{W}\right\}$ is generated from chromosomes $\mathcal{W}_{w} \in \Omega, w=1, \ldots, W$. Each chromosome represents a candidate solution set of the precoding vectors $(\mathcal{V})$ and power allocations $(\mathcal{P})$ to problem $\mathbf{P}_{1}$. A chromosome $\mathcal{W}_{w}$ contains $N$ gene-groups which represent the precoding vectors (with power scaling) for $N$ subchannels, i.e., $\left\{\mathbf{w}_{w, 1}, \ldots, \mathbf{w}_{w, N}\right\} \in \mathcal{W}_{w}$ where $\mathbf{w}_{w, n}$ is a candidate solution of $\sqrt{p_{n}} \mathbf{v}_{n}$. Each gene-group contains $M$ genes, where $M$ is the dimension of $\mathbf{w}_{w, n}$. Here, $\sqrt{p_{n}} \mathbf{v}_{n}$ is randomly generated following the constraints (7) and (8).

Evolution and proliferation: The (parent) chromosomes in the population will generate child chromosomes in the evolution and proliferation processes. The evolution process designs the precoding vectors by rotating the vector $\mathbf{w}_{w, n}$ in the $M$ dimensional space, while the proliferation process designs power allocation by altering $\left\|\mathbf{w}_{w, n}\right\|^{2}$ under the constraint $\sum_{n=1}^{N}\left\|\mathbf{w}_{w, n}\right\|^{2} \leq P$. In the evolution stage, first, for the $m$-th $(m=1, \ldots, M)$ gene of the $n$-th $(n=1, \ldots, N)$ gene-group from a parent-chromosome $\mathcal{W}_{w} \in \Omega$, we define the evolution probability $\varphi_{w, n, m}^{[\mathrm{e}]}$ and randomly generate a value from the interval $[0,1]$. If the generated value is smaller than $\varphi_{w, n, m}^{[\mathrm{e}]}$, then the $m$-th gene (and all genes in the $n$-th gene group) will go through the procedure of evolution; otherwise, the gene (or genes) will remain unchanged. Specifically, for the $m$-th gene of the $n$-th gene-group, we rotate the vector $\mathbf{w}_{w, n}$ toward the $m$-th axis in the $M$-dimensional space by an angle of $\theta$ or $-\theta$. The angles $\theta$ and $-\theta$ are selected equiprobably. If $\theta$ is selected, then after evolution the $n$-th gene-group is given by

$$
\mathbf{w}_{w, n}^{[\mathrm{e}]}=r_{1}(\theta) \mathbf{a}_{m}+r_{2}(\theta) \mathbf{w}_{w, n}
$$

where

$$
\begin{aligned}
r_{1}(\theta) & =\sqrt{\frac{\left\|\mathbf{w}_{w, n}\right\|^{4} \sin ^{2} \theta}{\left\|\mathbf{w}_{w, n}\right\|^{4}-\left(\mathbf{a}_{m}^{\top} \mathbf{w}_{w, n}\right)^{2}}} \\
r_{2}(\theta) & =\cos \theta-\sqrt{\frac{\sin ^{2} \theta}{\left\|\mathbf{w}_{w, n}\right\|^{4}-\left(\mathbf{a}_{m}^{\top} \mathbf{w}_{w, n}\right)^{2}}} \cdot \mathbf{a}_{m}^{\top} \mathbf{w}_{w, n} \\
\mathbf{a}_{m} & =[0, \ldots, 0, \underbrace{\left\|\mathbf{w}_{w, n}\right\|}_{\text {the } m \text {-th element }}, 0, \ldots, 0]^{\top} .
\end{aligned}
$$

Note that (13) and (14) are derived by solving the following two equations: 1) $\left\|r_{1}(\theta) \mathbf{a}_{m}+r_{2}(\theta) \mathbf{w}_{w, n}\right\|^{2}=\left\|\mathbf{w}_{w, n}\right\|^{2}$, and 2) $\left\|\mathbf{w}_{w, n}\right\|^{2} \cos \theta=\left(r_{1}(\theta) \mathbf{a}_{m}+r_{2}(\theta) \mathbf{w}_{w, n}\right)^{\top} \mathbf{w}_{w, n}$. If $-\theta$ is selected, the evolved gene-group can be expressed similarly by replacing $\theta$ with $-\theta$.

In the proliferation stage, the power allocation for the $N$ subchannels is determined. With the power constraint (7), the $n$-th $(n=1, \ldots, N)$ gene-group of the $l$-th $(l=1, \ldots, N)$ child-chromosome generated from the $w$-th $(w=1, \ldots, W)$ parent-chromosome can be expressed as

$$
\mathbf{w}_{w, l, n}^{[p]}=p_{w, l, n}^{[\mathrm{p}]} \frac{\mathbf{w}_{w, n}^{[\mathrm{e}]}}{\left\|\mathbf{w}_{w, n}^{[\mathrm{e}]}\right\|}
$$


where $p_{w, l, n}^{[\mathrm{p}]}=\min \left\{\left\|\mathbf{w}_{w, n}^{[\mathrm{e}]}\right\|+q, P\right\}$ for $l=n$ and $p_{w, l, n}^{[\mathrm{p}]}=$ $\max \left\{\left\|\mathbf{w}_{w, n}^{[\mathrm{e}]}\right\|-\bar{q}, 0\right\}$ for $l \neq n$, with $q$ and $\bar{q}$ being the power adjustment factor. Note that $\bar{q}$ is chosen with a given $q$ such that the power constraint $\sum_{n=1}^{N} p_{w, l, n}^{[\mathrm{p}]}=P$ is met. We denote $\mathcal{W}_{w, l}^{[\mathrm{p}]}=\left\{\mathbf{w}_{w, l, 1}^{[\mathrm{p}]}, \ldots, \mathbf{w}_{w, l, N}^{[\mathrm{p}]}\right\}$ as the $l$-th childchromosome generated from the $w$-th parent-chromosome, and $\Omega^{[\mathrm{p}]}=\left\{\mathcal{W}_{1,1}^{[\mathrm{p}]}, \ldots, \mathcal{W}_{1, N}^{[\mathrm{p}]}, \ldots, \mathcal{W}_{W, 1}^{[\mathrm{p}]}, \ldots, \mathcal{W}_{W, N}^{[\mathrm{p}]}\right\}$ as the population of all child-chromosomes after proliferation.

Mutation: The mutation process contributes to the increase in variation and diversity of the population to prevent premature convergence. For each gene-group in a child-chromosome, e.g., $\mathbf{w}_{w, l, n}^{[\mathrm{p}]} \in \mathcal{W}_{w, l}^{[\mathrm{p}]}$, we set a mutation probability $\varphi_{w, l, n}^{[\mathrm{m}]}$ to determine whether the genes require an irregular/tremendous change. ${ }^{1}$ The mutation probability is generally set much lower than the evolution probability. We denote the set of the updated child-chromosomes after mutation as $\Omega^{[\mathrm{m}]}=$ $\left\{\mathcal{W}_{1,1}^{[\mathrm{m}]}, \ldots, \mathcal{W}_{1, N}^{[\mathrm{m}]}, \ldots, \mathcal{W}_{W, 1}^{[\mathrm{m}]}, \ldots, \mathcal{W}_{W, N}^{[\mathrm{m}]}\right\}$

Evaluation: The evaluation process differentiates good chromosomes from bad chromosomes such that the population will evolve toward the Pareto optimal. The evaluation of chromosomes is based on the dominate-level of the chromosomes. We say that chromosome $\mathcal{W}_{i}$ has a higher dominate-level than chromosome $\mathcal{W}_{j}$ if chromosome $\mathcal{W}_{i}$ dominates chromosome $\mathcal{W}_{j}$ [24]. Chromosomes having the same dominate-level do not dominate each other. For a chromosome, we find $R_{n, k}$ ( $n=1, \ldots, N$ and $k=1, \ldots, K)$ and $\varepsilon_{n, k}$ from (2) and (4), respectively. The variables $\mathcal{A}$ can be determined by assigning the channels to the users who will gain the most data rate, i.e., $\alpha_{n, k^{*}}=1$ for which $k^{*}=\arg \max _{k} R_{n, k}, \forall n$, and otherwise $\alpha_{n, k}=0$. Then, the objectives $f_{1, k}(\mathcal{P}, \mathcal{V})$ and $f_{2, k}(\mathcal{A}, \mathcal{P}, \mathcal{V}), \forall k$ corresponding to this chromosome are obtained. By comparing the objectives corresponding to the $W(N+1)$ chromosomes in $\Omega \cup \Omega^{[\mathrm{m}]}$, we determine the dominate-levels of these chromosomes.

Selection: The selection process selects $W$ chromosomes with the largest dominate-levels from $\Omega \cup \Omega^{[\mathrm{m}]}$ to produce the next generation. We denote the set of the selected chromosomes by $\Omega^{[\mathrm{s}]}$. Then, update the population by $\Omega=\Omega^{[\mathrm{s}]}$.

Note that a genetic algorithm is usually said to converge when there is no significant improvement in the values of fitness of the population from one generation to the next [17]. In the proposed MOGA, a non-dominated chromosome persists for generations until it is dominated by other child chromosomes. Thus, with the growth of the number of iterations, the number of the non-dominated chromosomes with the largest dominate-level in a population will increase and the objectives corresponding to these non-dominated chromosomes will gradually approach to the Pareto front. Consequently, we will find a population with no child chromosome that dominates all of its parent chromosomes, in which case the MOGA converges.

\footnotetext{
${ }^{1}$ In this work, we update the mutated gene-group by a random vector under the constraint $\left\|\mathbf{w}_{w, l, n}^{[\mathrm{m}]}\right\|^{2}=p_{w, l, n}^{[\mathrm{p}]}$, where $\mathbf{w}_{w, l, n}^{[\mathrm{m}]}$ denotes the gene-group after mutation.
}

\begin{tabular}{l}
\hline Algorithm 2: MOGA \\
1 Initialization: Initialize a population $\Omega$. \\
2 Evolution: Evolve the genes in $\Omega$ according to (12). \\
3 Proliferation: Generate $N$ child-chromosomes from each \\
parent-chromosome according to (16). The generated \\
$W N$ child-chromosomes form a new population $\Omega^{[\mathrm{p}]}$. \\
4 Mutation: Mutate the genes in $\Omega^{[\mathrm{p}]}$ to yield a new \\
population $\Omega^{[\mathrm{m}]}$. \\
5 Evaluation: Determine the dominate-level for each \\
chromosome in $\Omega \cup \Omega^{[\mathrm{m}]}$. \\
6 Selection: Select $W$ chromosomes with the largest \\
dominate-levels from $\Omega \cup \Omega^{[\mathrm{m}]}$ to yield a new population \\
$\Omega^{[\mathrm{s}]}$. Let $\Omega=\Omega^{[\mathrm{s}]}$. \\
7 Repeat Steps $2-6$ until convergence, i.e., all solutions in \\
the $(i-1)$-th iteration are non-dominated by the \\
solutions in the $i$-th iteration, where $i>1$ is an integer. \\
\hline
\end{tabular}

\section{Simulation Results And Discussion}

Here, we show the numerical verification of SDR-WA and MOGA for solving an MOO problem with the objectives of maximizing the sum harvested power and the sum information rate. $^{2}$ The bandwidth is assumed to be $1 \mathrm{~Hz}$, which is uniformly divided into $N$ subchannels. The transmit power constraint is $P=1 \mathrm{~mW}(0 \mathrm{dBm})$. The path loss between the transmitter and each user is assumed to be $-100 \mathrm{~dB}$, i.e., $\sigma_{h}^{2}=10^{-10}$, and the noise variance is $\sigma_{z}^{2}=10^{-11.2} \mathrm{~mW}$ $(-112 \mathrm{dBm} / \mathrm{Hz})$.

\section{A. Convergence of the Proposed Algorithms}

Fig. 2(a) and Fig. 2(b) show the convergence of SDR-WA and MOGA, respectively, with $N=10, K=4$, and $M=$ 4. In Fig. 2(a), different points are obtained by varying $\beta$ (where $\beta_{1, k}=\beta$ and $\beta_{2, k}=1-\beta, \forall k \in\{1, \ldots, K\}$ ), after various numbers of iterations. As can be seen, more iterations push the non-dominated front a little further, with however diminishing returns as the number of iterations increases. Our experiments show that convergence is typically achieved in only few iterations (less than 5).

We set the configurations in MOGA as $(W, \theta, q)=$ $(100,10,0.01), \quad \varphi_{w, n, m}^{[\mathrm{e}]}=0.2, \forall w, n, m$, and $\varphi_{w, l, n}^{[\mathrm{m}]}=$ $0.05, \forall w, l, n$. Fig. 2(b) shows the objectives corresponding to all (parent) chromosomes in the population after 1,10 , 100 , and 1000 iterations. It is observed that initially the objective values are widely distributed in the lower left corner of the figure, and after several iterations the objective values gradually converge toward a greater non-dominated front. With 10 and 100 iterations, the objective values are more concentrated; however, the Pareto dominance among these objective values has wide disparity, i.e., chromosomes occupy numerous dominate-levels. As shown in Fig. 2(b), the search in the beginning is guided by the minority of non-dominated

\footnotetext{
${ }^{2}$ Note that our numerical verification focuses on a bi-objective simplification of problem $\mathbf{P}_{1}$ with objectives $\sum_{k=1}^{K} f_{1, k}$ and $\sum_{k=1}^{K} f_{2, k}$ merely for convenience of illustration.
} 


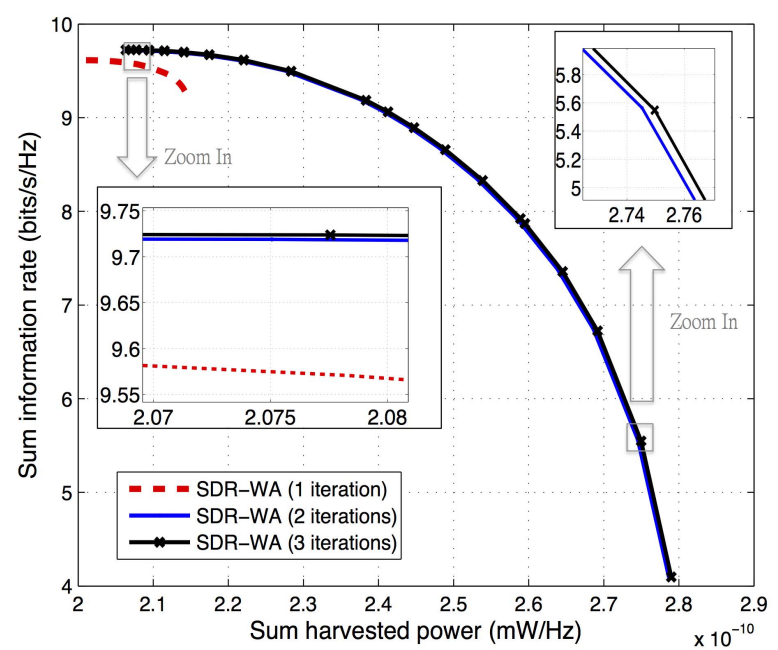

(a)

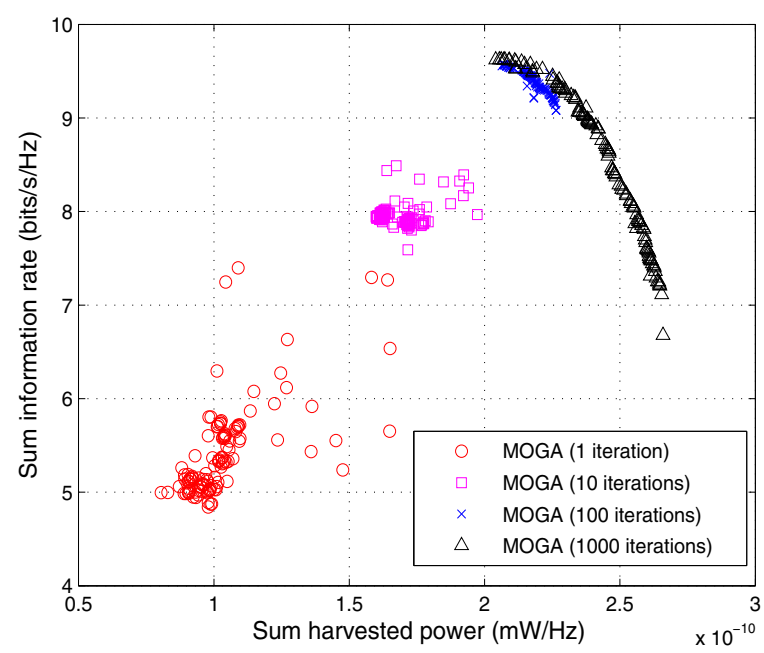

(b)

Fig. 2. Achievable sum information rate and sum harvested power in a downlink multiuser OFDMA system by using (a) SDR-WA and (b) MOGA methods, where $N=10, K=4$, and $M=4$.

solutions such that all candidate solutions are close to each other. After 1000 iterations, there is almost no single child chromosome that dominates all other parent chromosomes. Thus, the objective values are distributed more widely, with most chromosomes mutually non-dominated with each other.

\section{B. Tradeoff between Information Rate and Harvested Power}

Fig. 3 compares the tradeoffs of the sum information rate vs. the sum harvested power for SDR-WA (10 iterations) and MOGA (1000 iterations). For the same system configuration, MOGA slightly outperforms SDR-WA, with increasing advantages as $K, M$, or $N$ increases. Increasing $K$ results in a greater sum harvested power for the same sum information rate, as shown in Fig. 3(a), because there are more users who could harvest power from the environment. Increasing $N$ results in a greater sum harvested power, as can be seen from (5) and verified in Fig. 3(b). Theoretically, increasing either
$K$ or $N$ will also lead to a higher sum information rate due to the diversity gain. This can be exemplified by comparing sufficiently different values of $K$ or $N$ and is not particularly illustrated in Fig. 3. Increasing $M$ slightly however leads to noticeable increases in both the sum harvest power and sum information rate, as shown in Fig. 3(b). This is due to the better beamforming capability at the transmitter with more antennas.

Verifying that a genetic algorithm has converged to a global optimum for an NP-hard problem is still an open issue, unless a solution in the Pareto optimal set is already known. The SDR technique has been shown to provide a good approximation to the global optimum [21]. Fig. 3 shows that SDR-WA and MOGA converge to solutions that are very close, suggesting that the solutions yielded by MOGA are also close to the optimal solution.

\section{Comparison of Complexity}

Here, we analyze the complexity of the proposed SDRWA and MOGA algorithms. Given the information about the weights of all objectives, the SDR-WA can find a solution in only few iterations. The computational complexity of SDRWA in each iteration is dominated by the process of finding the Lagrange multiplier $\mu$ in (11), which is given by $O\left(N \log _{2} \Upsilon\right)$, where $\Upsilon$ is the number of equal-partitions of the searching interval of Lagrangian multiplier $\mu$, with the partition based on the accuracy tolerance [25, Chapter 2]. SDR-WA may require additional computations for searching the combining weights of objectives. As compared to SDR-WA, MOGA can simultaneously produce $W$ solutions selected from a set of $W N$ candidate solutions in each iteration, where the computational complexity is given by $O(W N)$. Since $\log _{2} \Upsilon \gg W$ in general, MOGA has lower complexity than SDR-WA in each iteration. However, to solve the MOO problem $\mathbf{P}_{1}$, MOGA requires more iterations to achieve convergence. While a comprehensive comparison may be difficult, our experiments show that SDR-WA has a lower computational complexity (in terms of simulation run time) than MOGA when the number of objectives in the considered MOO problem is small, as in all cases of configurations in Fig. 3.

\section{CONCLUSION}

This work formulates the resource allocation problem for SWIPT in a downlink multiuser OFDMA system as an MOO problem with $2 K$ conflicting objectives, i.e., maximizing the information rate and the harvested power for $K$ users. Solving the MOO problem presents a joint design of subchannel allocation, power allocation, and beamforming, which is difficult due to nonconvexity. Two methods, SDR-WA and MOGA, are proposed to solve the MOO problem. The SDR-WA method is efficient when there is prior information about the objectives, while the MOGA method may achieve a better result if a sufficient number of iterations takes place. Simulation shows the convergence and validity of both proposed methods. The obtained achievable Pareto optimal solution set illustrates the tradeoffs of the sum information rate vs. the sum harvested power in the considered system. The harvested power, in the 


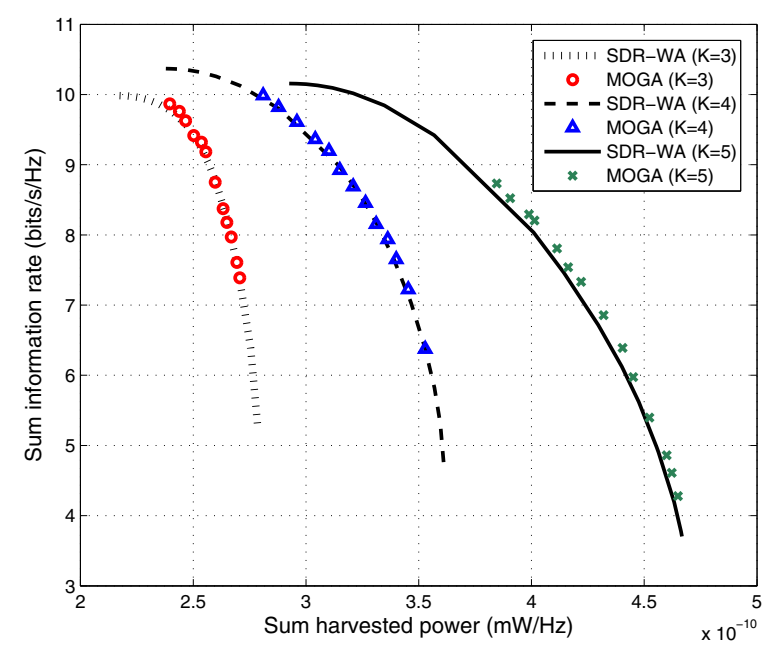

(a)

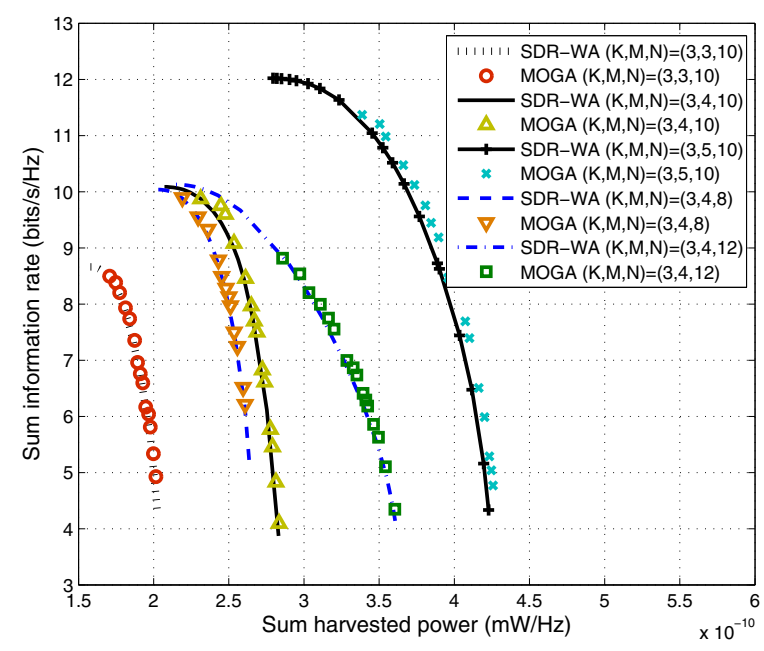

(b)

Fig. 3. Comparison of the achievable sum information rate vs. sum harvested power in a downlink multiuser OFDMA system, with (a) fixed $M=4$, $N=10$, and varying $K$, and (b) fixed $K=3$ and varying $M, N$.

order of $10^{-10} \mathrm{~mW} / \mathrm{Hz}$, can be nontrivial to address the energy shortage problem in future millimeter wave communications with broad bandwidth.

\section{REFERENCES}

[1] Y. Chen, F. Han, Y.-H. Yang, H. Ma, Y. Han, C. Jiang, H.-Q. Lai, D. Claffey, Z. Safar, and K. J. R. Liu, "Time-reversal wireless paradigm for green internet of things: an overview," IEEE Internet Things J., vol. 1, no. 1, pp. 81-98, Feb. 2014.

[2] L. Atzori, A. Iera, and G. Morabito, "The internet of things: a survey," Comput. Netw., vol. 54, no. 15, pp. 2787-2805, Oct. 2010.

[3] M. Gregori and M. Payaró, "Energy-efficient transmission for wireless energy harvesting nodes," IEEE Trans. Wireless Commun., vol. 12, no. 3, pp. 1244-1254, March 2013.

[4] X. Lu, P. Wang, D. Niyato, D. I. Kim, and Z. Han, "Wireless networks with RF energy harvesting: A contemporary survey," Commun. Surveys Tuts., 2015.
[5] D. W. K. Ng, E. S. Lo, and R. Schober, "Energy efficient power allocation in OFDM systems with wireless information and power transfer," in IEEE ICC Selected Areas in Communications (SAC) Symposium, Budapest, June 2013, pp. 4125-4130.

[6] K. Tutuncuoglu and A. Yener, "Optimum transmission policies for battery limited energy harvesting nodes," IEEE Trans. Wireless Commun., vol. 11, no. 3, pp. 1180-1189, March 2012.

[7] M. Zafer and E. Modiano, "A calculus approach to energy-efficient data transmission with quality-of-service constraints," IEEE/ACM Trans. on Netw., vol. 17, no. 3, pp. 898-911, June 2009.

[8] L. Varshney, "Transporting information and energy simultaneously," in IEEE International Symposium on Information Theory Proceedings (ISIT), Toronto, Canada, June 2008, pp. 1612-1616.

[9] P. Grover and A. Sahai, "Shannon meets Tesla: Wireless information and power transfer," in IEEE International Symposium on Information Theory Proceedings (ISIT), Austin, TX, June 2010, pp. 2363 -2367.

[10] J. Xu, L. Liu, and R. Zhang, "Multiuser MISO beamforming for stimultaneous wireless information and power transfer," in IEEE International Conference on Acoustics, Speech, and Signal Processing (ICASSP), Vancouver, BC, May 2013, pp. 4754-4758.

[11] R. Zhang and C. K. Ho, "MIMO broadcasting for simultaneous wireless information and power transfer," IEEE Trans. Wireless Commun., vol. 12, no. 5, pp. 1989-2001, May 2013.

[12] X. Zhou, R. Zhang, and C. K. Ho, "Wireless information and power transfer in multiuser OFDM systems," in IEEE Global Telecommunications Conference (GLOBECOM), Atlanta, GA, USA, Sept. 2013, pp. 4092-4097.

[13] W. Wang, L. Li, Q. Sun, and J. Jin, "Power allocation in multiuser MIMO systems for simultaneous wireless information and power transfer,", in IEEE Vehicular Technology Conference (VTC Fall), Las Vegas, NV, USA, Sept. 2013, pp. 1-5.

[14] K. Huang and E. Larsson, "Simultaneous information and power transfer for broadband wireless systems," IEEE Trans. Signal Processing, vol. 61, no. 23, pp. 5972-5986, Dec. 2013.

[15] J. Park and B. Clerckx, "Joint wireless information and energy transfer in a $k$-user MIMO interference channel." IEEE Trans. Wireless Commun., vol. 13, no. 10, pp. 5781-5796, July 2014.

[16] P. Ngatchou, A. Zarei, and M. El-Sharkawi, "Pareto multi objective optimization," in Proceedings of the 13th International Conference on Intelligent Systems Application to Power Systems, Arlington, VA, Nov. 2005, pp. 84-91.

[17] K. Deb, Multi-Objective Optimization using Evolutionary Algorithms. Wiley, 2004, vol. 16.

[18] H.-J. Chou, C.-J. Tsao, J.-M. Wu, J.-Y. Hsu, and P.-A. Ting, "Fairness based resource allocation for multiuser MIMO communication networks," in IEEE Global Telecommunications Conference (GLOBECOM), Austin, TX, USA, Dec. 2014.

[19] N. Zhao, F. Yu, and V. Leung, "Wireless energy harvesting in interference alignment networks," IEEE Communications Magazine, vol. 53, no. 6, pp. 72-78, June 2015.

[20] I. Csiszar and G. Tusnady, "Information geometry and alternating minimization procedures," Statist. Decisions, vol. 1, pp. 205-237, 1984.

[21] S. Boyd and L. Vandenberghe, Convex Optimization. Cambridge University Press, 2004.

[22] C. Helmberg, F. Rendl, R. J. Vanderbei, and H. Wolkowicz, An InteriorPoint Method for Semidefinite Programming, 2nd ed. Program in Statistics and Operations Research, Princeton University, Jan. 2005.

[23] Z. Wen, D. Goldfarb, and W. Yin, "Alternating direction augmented Lagrangian methods for semidefinite programming," Springer and Mathematical Optimization Society, vol. 2, no. 3-4, pp. 203-230, Sept. 2010.

[24] C. Lin and B.-S. Chen, "Achieving pareto optimal power tracking control for interference limited wireless systems via multi-objective $\mathrm{h} 2 / \mathrm{h} \infty$ optimization," IEEE Trans. Wireless Commun., vol. 12, no. 12, pp. 61546165, Dec. 2013

[25] C. W. Chen, Z. Li, and S. Lian, Intelligent Multimedia Communication: Techniques and Applications, 1, Ed. Springer-Verlag Berlin Heidelberg, 2011. 